\title{
Solvability for a Coupled System of Fractional Integrodifferential Equations with $m$-Point Boundary Conditions on the Half-Line
}

\author{
Payam Nasertayoob, ${ }^{1}$ S. Mansour Vaezpour, ${ }^{1}$ and Dumitru Baleanu' ${ }^{2}$ \\ ${ }^{1}$ Department of Mathematics, Amirkabir University of Technology, Hafez Avenue, P.O. Box 15914, Tehran 15875-4413, Iran \\ ${ }^{2}$ Department of Mathematics and Computer Sciences, Faculty of Arts and Sciences, Cankaya University, 06530 Ankara, Turkey
}

Correspondence should be addressed to S. Mansour Vaezpour; vaez@aut.ac.ir

Received 20 May 2014; Accepted 1 July 2014; Published 23 July 2014

Academic Editor: Guo-Cheng Wu

Copyright (C) 2014 Payam Nasertayoob et al. This is an open access article distributed under the Creative Commons Attribution License, which permits unrestricted use, distribution, and reproduction in any medium, provided the original work is properly cited.

The aim of this paper is to study the solvability for a coupled system of fractional integrodifferential equations with multipoint fractional boundary value problems on the half-line. An example is given to demonstrate the validity of our assumptions.

\section{Introduction}

The theory of derivatives and integrals of fractional order has undergone rapid development over the years and played a very important role in modern applied mathematical models of real processes arising in phenomena studied in physics, mechanics, engineering, and so on [1-3]. Recently, the existence of solutions for coupled systems involving fractional differential equations is one of the theoretical fields investigated by many authors [4-13].

Very recently, Wang et al. [10] studied the existence of solutions for the following coupled system of nonlinear fractional differential equations by using Schauder's fixed point theorem:

$$
\begin{array}{cc}
D^{p} u(t)+f(t, v(t))=0, & 2<p<3, t \in J:=[0, \infty), \\
D^{q} v(t)+g(t, u(t))=0, & 2<q<3, t \in J:=[0, \infty), \\
u(0)=u^{\prime}(0)=0, & D^{p-1} u(\infty)=\sum_{i=1}^{m-2} \beta_{i} u\left(\xi_{i}\right), \\
v(0)=v^{\prime}(0)=0, & D^{q-1} v(\infty)=\sum_{i=1}^{m-2} \gamma_{i} v\left(\xi_{i}\right),
\end{array}
$$

where $f, g \in C(J \times \mathbb{R}, \mathbb{R}), 0<\xi_{1}<\xi_{2}<\cdots<\xi_{m-2}<\infty$, and $D^{p}$ and $D^{q}$ denote Riemann-Liouville fractional derivatives of order $p$ and order $q$, respectively; also $\beta_{i}>0, \gamma_{i}>0$ are such that $0<\sum_{i=1}^{m-2} \beta_{i} \xi_{i}^{p-1}<\Gamma(p)$ and $0<\sum_{i=1}^{m-2} \gamma_{i} \xi_{i}^{q-1}<$ $\Gamma(q)$.

Motivated by [10], in this paper, we consider a coupled system of nonlinear fractional integrodifferential equations on an unbounded domain and more general boundary conditions:

$$
\begin{gathered}
D^{\alpha} u(t)+f\left(t, v(t), I^{\alpha} v(t)\right)=0, \quad t \in J=[0, \infty), \\
D^{\beta} v(t)+g\left(t, u(t), I^{\beta} u(t)\right)=0, \quad t \in J=[0, \infty), \\
u(0)=u^{\prime}(0)=0, \quad v(0)=v^{\prime}(0)=0, \\
D^{\alpha-1} u(\infty)=\sum_{i=1}^{m-2} a_{i} u\left(\xi_{i}\right)+\sum_{i=1}^{m-2} b_{i} D^{\alpha-1} u\left(\xi_{i}\right), \\
D^{\beta-1} v(\infty)=\sum_{i=1}^{m-2} c_{i} v\left(\xi_{i}\right)+\sum_{i=1}^{m-2} d_{i} D^{\beta-1} v\left(\xi_{i}\right),
\end{gathered}
$$

where $2<\alpha, \beta \leq 3,0<\xi_{1}<\xi_{2}<\cdots<\xi_{m-2}<\infty$, $a_{i}, b_{i}, c_{i}, d_{i} \geq 0$ are real numbers, $f, g \in C(J \times \mathbb{R} \times \mathbb{R}, \mathbb{R})$, and Ddenotes Riemann-Liouville fractional derivative. It is clear that boundary value problem (2) includes problem (1) as special case. 
Integrodifferential equations have become important in recent years as mathematical models of phenomena in both the physical and social sciences. In particular, some physical phenomena involving certain type of memory effects are represented by integrodifferential equations [14-18].

However, to the best of our knowledge, no work has been reported on the existence results for coupled system of nonlinear fractional integrodifferential equations on an unbounded domain.

The paper is organized as follows. In Section 2, we recall some basic definitions, notations, and preliminary facts. Section 3 is devoted to the existence results for system of nonlinear fractional integrodifferential equations on an unbounded domain. In Section 4, an example is given to demonstrate the applicability of our results.

\section{Preliminaries}

In this section, we will first recall some basic definitions and lemmas which are used in what follows and can be found in $[2,19]$.

Definition 1. The Riemann-Liouville fractional integral of order $\delta>0$ of a function $f:(0, \infty) \rightarrow \mathbb{R}$ is given by

$$
I^{\delta} f(t)=\frac{1}{\Gamma(\delta)} \int_{0}^{t}(t-s)^{\delta-1} f(s) d s,
$$

provided that the right-hand side is pointwise defined.

Definition 2. The Riemann-Liouville fractional derivative of order $\delta>0$ of a continuous function $f:(0, \infty) \rightarrow \mathbb{R}$ is given by

$$
D^{\delta} f(t)=\frac{1}{\Gamma(n-\delta)}\left(\frac{d}{d t}\right)^{n} \int_{0}^{t}(t-s)^{n-\delta-1} f(s) d s,
$$

where $n=[\alpha]+1$, provided that the right-hand side is pointwise defined.

Remark 3. The following properties are well known:

$$
\begin{gathered}
D^{\delta} I^{\delta} f(t)=f(t), \quad \delta>0, \quad f(t) \in L^{1}(0, \infty), \\
D^{\alpha} I^{\delta} f(t)=I^{\delta-\alpha} f(t), \quad \delta>\alpha>0, f(t) \in L^{1}(0, \infty), \\
D^{\delta} t^{\lambda}=\frac{\Gamma(\lambda+1)}{\Gamma(\lambda-\delta+1)} t^{\lambda-\delta}, \quad \lambda>-1, t>0 .
\end{gathered}
$$

Lemma 4. For $\delta>0$, the equation $D^{\delta} u(t)=0$ is valid if and only if

$$
\begin{array}{r}
u(t)=c_{1} t^{\delta-1}+c_{2} t^{\delta-2}+\cdots+c_{n} t^{\delta-n}, \\
c_{i} \in \mathbb{R}, \quad j=1,2, \ldots, n,
\end{array}
$$

where $n$ is the smallest integer greater than or equal to $\delta$.

Lemma 5. Assume that $D^{\delta} u(t) \in L^{1}(0, \infty)$; then,

$$
\begin{array}{r}
I^{\delta} D^{\delta} u(t)=u(t)+c_{1} t^{\delta-1}+c_{2} t^{\delta-2}+\cdots+c_{n} t^{\delta-n}, \\
c_{i} \in \mathbb{R}, \quad j=1,2, \ldots, n,
\end{array}
$$

where $n$ is the smallest integer greater than or equal to $\delta$.
For any $\delta>1$ we can define the space

$$
X_{\delta}=\left\{u \in C[0, \infty): \sup _{t \in J} \frac{|u(t)|}{1+t^{\delta-1}}<\infty\right\},
$$

equipped with the norm

$$
\|u\|_{\delta}=\sup _{t \in J} \frac{|u(t)|}{1+t^{\delta-1}} .
$$

Clearly, $\left(X_{\delta},\|u\|_{\delta}\right)$ is a Banach space [19]. For $(u, v) \in X_{\alpha} \times X_{\beta}$ we define

$$
\|(u, v)\|_{\alpha, \beta}=\max \left\{\|u\|_{\alpha},\|v\|_{\beta}\right\} ;
$$

then, $\left(X_{\alpha} \times X_{\beta},\|\cdot\|_{\alpha, \beta}\right)$ is a Banach space.

\section{Main Results}

In this section, we prove the existence results for the boundary value problem (2). For convenience we use the following notation:

$$
\Delta_{\alpha}=\Gamma(\alpha)-\sum_{i=1}^{m-2} a_{i} \xi_{i}^{\alpha-1}-\Gamma(\alpha) \sum_{i=1}^{m-2} b_{i} .
$$

By replacing $\alpha, a_{i}, b_{i}$ with $\beta, c_{i}, d_{i}$, respectively, we can define $\Delta_{\beta}$.

Lemma 6. Let $h \in C[0, \infty)$ and $\Delta_{\alpha}>0$; then, the unique solution of

$$
\begin{aligned}
& D^{\alpha} u(t)+h(t)=0, \quad 2<\alpha<3, t \in J=[0, \infty), \\
& u(0)=u^{\prime}(0)=0, \\
& D^{\alpha-1} u(\infty)=\sum_{i=1}^{m-2} a_{i} u\left(\xi_{i}\right)+\sum_{i=1}^{m-2} b_{i} D^{\alpha-1} u\left(\xi_{i}\right)
\end{aligned}
$$

is given by

$$
u(t)=\int_{0}^{\infty} G_{\alpha}(t, s) h(s) d s,
$$

where $G_{\alpha}(t, s)$ is Green's function given by

$$
\begin{gathered}
G_{\alpha}(t, s)=K_{\alpha}(t, s)+\frac{t^{\alpha-1}}{\Delta_{\alpha}} \sum_{i=1}^{m-2} a_{i} K_{\alpha}\left(\xi_{i}, s\right) \\
+\frac{t^{\alpha-1}}{\Delta_{\alpha}} \sum_{i=1}^{m-2} b_{i} H\left(\xi_{i}, s\right),
\end{gathered}
$$

with

$$
\begin{gathered}
K_{\alpha}(t, s) \\
=\frac{1}{\Gamma(\alpha)} \begin{cases}t^{\alpha-1}-(t-s)^{\alpha-1}, & 0 \leq s \leq t<\infty, \\
t^{\alpha-1}, & 0 \leq t \leq s<\infty,\end{cases} \\
H(t, s)= \begin{cases}0, & 0 \leq s \leq t<\infty, \\
1, & 0 \leq t \leq s<\infty .\end{cases}
\end{gathered}
$$


Proof. By Lemma 5, the solution of (12) can be writen as

$$
u(t)=c_{1} t^{\alpha-1}+c_{2} t^{\alpha-2}+c_{3} t^{\alpha-3}-I^{\alpha} h(t)
$$

Using the boundary conditions (13), we find that $c_{2}=c_{3}=0$ and

$$
D^{\alpha-1} u(t)=c_{1} \Gamma(\alpha)-I^{1} h(t) .
$$

Now considering the second boundary condition, we have

$$
\begin{aligned}
& c_{1}=\frac{1}{\Delta_{\alpha}}\left(\int_{0}^{\infty} h(s) d s\right. \\
& \\
&-\frac{\sum_{i=1}^{m-2} a_{i}}{\Gamma(\alpha)} \int_{0}^{\xi_{i}}\left(\xi_{i}-s\right)^{\alpha-1} h(s) d s \\
&\left.-\sum_{i=1}^{m-2} b_{i} \int_{0}^{\xi_{i}} h(s) d s\right) .
\end{aligned}
$$

Therefore, the unique solution of the boundary value problem (12)-(14) is

$$
\begin{aligned}
& u(t)=\frac{t^{\alpha-1}}{\Delta_{\alpha}}\left(\int_{0}^{\infty} h(s) d s\right. \\
& -\frac{\sum_{i=1}^{m-2} a_{i}}{\Gamma(\alpha)} \int_{0}^{\xi_{i}}\left(\xi_{i}-s\right)^{\alpha-1} h(s) d s \\
& \left.-\sum_{i=1}^{m-2} b_{i} \int_{0}^{\xi_{i}} h(s) d s\right) \\
& -\frac{1}{\Gamma(\alpha)} \int_{0}^{t}(t-s)^{\alpha-1} h(s) d s \\
& =\int_{0}^{\infty} K_{\alpha}(t, s) h(s) d s \\
& +\frac{\sum_{i=1}^{m-2} a_{i} t^{\alpha-1}}{\Delta_{\alpha}} \int_{0}^{\infty} \frac{\xi_{i}^{\alpha-1}}{\Gamma(\alpha)} h(s) d s \\
& +\frac{\Gamma(\alpha) \sum_{i=1}^{m-2} b_{i} t^{\alpha-1}}{\Delta_{\alpha}} \int_{0}^{\infty} \frac{h(s)}{\Gamma(\alpha)} d s \\
& -\frac{\sum_{i=1}^{m-2} a_{i} t^{\alpha-1}}{\Delta_{\alpha}} \int_{0}^{\xi_{i}} \frac{\left(\xi_{i}-s\right)^{\alpha-1}}{\Gamma(\alpha)} h(s) d s \\
& -\frac{\Gamma(\alpha) \sum_{i=1}^{m-2} b_{i} t^{\alpha-1}}{\Delta_{\alpha}} \int_{0}^{\xi_{i}} \frac{h(s)}{\Gamma(\alpha)} d s \\
& =\int_{0}^{\infty} K_{\alpha}(t, s) h(s) d s \\
& +\frac{\sum_{i=1}^{m-2} a_{i} t^{\alpha-1}}{\Delta_{\alpha}} \int_{0}^{\infty} K_{\alpha}\left(\xi_{i}, s\right) h(s) d s
\end{aligned}
$$

$$
\begin{aligned}
& +\frac{\sum_{i=1}^{m-2} b_{i} t^{\alpha-1}}{\Delta_{\alpha}} \int_{0}^{\infty} H\left(\xi_{i}, s\right) h(s) d s \\
= & \int_{0}^{\infty} G_{\alpha}(t, s) h(s) d s,
\end{aligned}
$$

where $G_{\alpha}(t, s), K_{\alpha}(t, s)$, and $H(t, s)$ are defined by (16), (17), and (18), respectively. The proof is complete.

Now, we introduce the following function:

$$
\begin{gathered}
G_{\beta}(t, s)=K_{\beta}(t, s)+\frac{t^{\beta-1}}{\Delta_{\beta}} \sum_{i=1}^{m-2} c_{i} K_{\beta}\left(\xi_{i}, s\right) \\
+\frac{t^{\beta-1}}{\Delta_{\beta}} \sum_{i=1}^{m-2} d_{i} H\left(\xi_{i}, s\right),
\end{gathered}
$$

where

$$
\begin{aligned}
& K_{\beta}(t, s) \\
& \quad=\frac{1}{\Gamma(\beta)} \begin{cases}t^{\beta-1}-(t-s)^{\beta-1}, & 0 \leq s \leq t<\infty, \\
t^{\beta-1}, & 0 \leq t \leq s<\infty .\end{cases}
\end{aligned}
$$

Remark 7. From the definition of $G_{\alpha}(t, s)$ and $G_{\beta}(t, s)$, for any $(s, t) \in[0, \infty) \times[0, \infty)$, we have

$$
\frac{G_{\alpha}(t, s)}{1+t^{\alpha-1}} \leq Q, \quad \frac{G_{\beta}(t, s)}{1+t^{\beta-1}} \leq Q,
$$

where

$$
\begin{aligned}
Q=\max \left\{\frac{1}{\Gamma(\alpha)}+\frac{\sum_{i=1}^{m-2} a_{i} \xi_{m-2}^{\alpha-1}}{\Gamma(\alpha) \Delta_{\alpha}}+\frac{\sum_{i=1}^{m-2} b_{i}}{\Delta_{\alpha}},\right. \\
\\
\left.\frac{1}{\Gamma(\beta)}+\frac{\sum_{i=1}^{m-2} c_{i} \xi_{m-2}^{\beta-1}}{\Gamma(\beta) \Delta_{\beta}}+\frac{\sum_{i=1}^{m-2} d_{i}}{\Delta_{\beta}}\right\} .
\end{aligned}
$$

Let an operator $T: X_{\alpha} \times X_{\beta} \rightarrow X_{\alpha} \times X_{\beta}$ be defined by

$$
\begin{aligned}
T(u, v)= & \left(T_{1}(v), T_{2}(u)\right) \\
= & \left(\int_{0}^{\infty} G_{\alpha}(t, s) f\left(s, v(s), I^{\alpha} v(s)\right) d s,\right. \\
& \left.\quad \int_{0}^{\infty} G_{\beta}(t, s) g\left(s, u(s), I^{\beta} u(s)\right) d s\right) .
\end{aligned}
$$

From the definition of operator $T$, the problem (2) has a solution if and only if the operator $T$ has a fixed point.

Theorem 8. Assume the following.

$\left(H_{1}\right)$ There exist nonnegative functions $a(t), b(t), \phi(t) \in$ $C[0, \infty)$ such that

$$
\begin{gathered}
|f(t, x, y)| \leq a(t)|x|+b(t)|y|+\phi(t), \\
\int_{0}^{\infty} \phi(t) d t<\infty, \quad \Delta_{\alpha}>0,
\end{gathered}
$$




$$
\begin{aligned}
\int_{0}^{\infty} & \left(1+t^{\alpha-1}\right) a(t) d t \\
& +\frac{1}{\Gamma(\alpha)} \int_{0}^{\infty}\left[\frac{t^{\alpha}}{\alpha}+B(\alpha, \alpha) t^{2 \alpha-1}\right] b(t) d t \\
& <\frac{1}{Q}
\end{aligned}
$$

where $B(\alpha, \alpha)$ is the beta-function.

$\left(H_{2}\right)$ There exist nonnegative functions $c(t), d(t), \varphi(t) \quad \epsilon$ $C[0, \infty)$ such that

$$
\begin{gathered}
|g(t, x, y)| \leq c(t)|x|+d(t)|y|+\varphi(t), \\
\int_{0}^{\infty} \varphi(t) d t<\infty, \quad \Delta_{\beta}>0, \\
\int_{0}^{\infty}\left(1+t^{\beta-1}\right) c(t) d t \\
+\frac{1}{\Gamma(\beta)} \int_{0}^{\infty}\left[\frac{t^{\beta}}{\beta}+B(\beta, \beta) t^{2 \beta-1}\right] d(t) d t \\
<\frac{1}{Q},
\end{gathered}
$$

where $B(\beta, \beta)$ is the beta-function.

Then, the system (2) has a solution.

Proof. Take

$$
\begin{aligned}
R>\max \{ & \left(Q \int_{0}^{\infty} \phi(t) d t\right) \\
& \times\left(1-Q \int_{0}^{\infty}\left(1+t^{\alpha-1}\right) a(t) d t-\frac{Q}{\Gamma(\alpha)}\right. \\
& \left.\times \int_{0}^{\infty}\left[\frac{t^{\alpha}}{\alpha}+B(\alpha, \alpha) t^{2 \alpha-1}\right] b(t) d t\right)^{-1}, \\
& \left(Q \int_{0}^{\infty} \varphi(t) d t\right) \\
& \times\left(1-Q \int_{0}^{\infty}\left(1+t^{\beta-1}\right) c(t) d t-\frac{Q}{\Gamma(\beta)}\right. \\
& \left.\left.\times \int_{0}^{\infty}\left[\frac{t^{\beta}}{\beta}+B(\beta, \beta) t^{2 \beta-1}\right] d(t) d t\right)^{-1}\right\}
\end{aligned}
$$

and define a ball

$$
B_{R}=\left\{(u, v) \in X_{\alpha} \times X_{\beta}:\|(u, v)\|_{\alpha, \beta} \leq R\right\} .
$$

At the first step, we prove that the operator $T$ transforms the ball $B_{R}$ into itself. For any $(u, v) \in B_{R}$ we have

$$
\begin{aligned}
& \left\|T_{1}(v)\right\|_{\alpha} \\
& \quad=\sup _{t \in J} \frac{1}{1+t^{\alpha-1}}\left|\int_{0}^{\infty} G_{\alpha}(t, s) f\left(s, v(s), I^{\alpha} v(s)\right) d s\right|
\end{aligned}
$$

$\leq \sup _{t \in J} \frac{1}{1+t^{\alpha-1}}$

$\times \int_{0}^{\infty} G_{\alpha}(t, s)\left[a(s)|v(s)|+b(s)\left|I^{\alpha} v(s)\right|+\phi(s)\right] d s$

$\leq Q \int_{0}^{\infty} a(s)|v(s)| d s+\frac{Q}{\Gamma(\alpha)}$

$\times \int_{0}^{\infty} b(s)\left[\int_{0}^{s}(s-\tau)^{\alpha-1} v(\tau) d \tau\right] d s$

$+Q \int_{0}^{\infty} \phi(s) d s$

$\leq Q\|v\|_{\alpha} \int_{0}^{\infty}\left(1+s^{\alpha-1}\right) a(s) d s$

$+\frac{Q\|v\|_{\alpha}}{\Gamma(\alpha)} \int_{0}^{\infty} b(s)\left[\int_{0}^{s}\left(1+\tau^{\alpha-1}\right)(s-\tau)^{\alpha-1} d \tau\right] d s$

$+Q \int_{0}^{\infty} \phi(s) d s$

$\leq Q\|v\|_{\alpha} \int_{0}^{\infty}\left(1+s^{\alpha-1}\right) a(s) d s$

$+\frac{Q\|v\|_{\alpha}}{\Gamma(\alpha)} \int_{0}^{\infty} b(s)\left[s^{\alpha} \int_{0}^{1}(1-\theta)^{\alpha-1}+s^{2 \alpha-1}\right.$

$\left.\times \int_{0}^{1} \theta^{\alpha-1}(1-\theta)^{\alpha-1} d \theta\right] d s$

$+Q \int_{0}^{\infty} \phi(s) d s$

$\leq Q\|v\|_{\alpha} \int_{0}^{\infty}\left(1+s^{\alpha-1}\right) a(s) d s$

$+\frac{Q\|v\|_{\alpha}}{\Gamma(\alpha)} \int_{0}^{\infty} b(s)\left[\frac{s^{\alpha}}{\alpha}+B(\alpha, \alpha) s^{2 \alpha-1}\right] d s$

$+Q \int_{0}^{\infty} \phi(s) d s<R$.

In a similar way, we can get

$\left\|T_{2}(u)\right\|_{\beta}$

$$
\begin{aligned}
\leq & Q\|u\|_{\beta} \int_{0}^{\infty}\left(1+s^{\beta-1}\right) c(s) d s \\
& +\frac{Q\|u\|_{\beta}}{\Gamma(\beta)} \int_{0}^{\infty} d(s)\left[\frac{s^{\beta}}{\beta}+B(\beta, \beta) s^{2 \beta-1}\right] d s \\
& +Q \int_{0}^{\infty} \varphi(t) d s<R .
\end{aligned}
$$

Hence, $\|T(u, v)\|_{\alpha, \beta} \leq R$ and this shows that $T B_{R} \subset B_{R}$. 
Next, we show that $T: B_{R} \rightarrow B_{R}$ is completely continuous. First, Let $\left(u_{n}, v_{n}\right) \rightarrow(u, v)$ as $n \rightarrow \infty$ in $B_{R}$. From (32) we have

$$
\int_{0}^{\infty}\left|f\left(s, v(s), I^{\alpha} v(s)\right)\right| d s<\frac{R}{Q} .
$$

Then, by the Lebsegue dominated convergence theorem and continuity of $f$, we obtain

$$
\begin{aligned}
& \int_{0}^{\infty} f\left(s, v_{n}(s), I^{\alpha} v_{n}(s)\right) d s \\
& \quad \longrightarrow \int_{0}^{\infty} f\left(s, v(s), I^{\alpha} v(s)\right) d s
\end{aligned}
$$

as $n \rightarrow \infty$. Therefore, by Remark 7, we have

$$
\begin{aligned}
& \left\|T_{1}\left(v_{n}\right)-T_{1}(v)\right\|_{\alpha} \\
& \leq Q \mid \int_{0}^{\infty} f\left(s, v_{n}(s), I^{\alpha} v_{n}(s)\right) d s \\
& \quad-\int_{0}^{\infty} f\left(s, v(s), I^{\alpha} v(s)\right) d s \mid \longrightarrow 0,
\end{aligned}
$$

as $n \rightarrow \infty$. Similar process can be repeated for $T_{2}$; thus, operator $T$ is continuous.

Now, we show that $T: B_{R} \rightarrow B_{R}$ is equicontinuous operator. Let $L>0$ and $t_{1}, t_{2} \in[L, \infty)$; without loss of generality, we may assume that $t_{1}<t_{2}$. Since $K_{\alpha}\left(\xi_{i}, s\right) \leq$ $\xi_{m-2}^{\alpha-1} / \Gamma(\alpha)$ and $H\left(\xi_{i}, s\right) \leq 1$, for any $s>0$ and $i=1,2, \ldots, m-$ 2 , we have

$$
\begin{aligned}
& \left|\frac{T_{1}(u)\left(t_{2}\right)}{1+t_{2}^{\alpha-1}}-\frac{T_{1}(u)\left(t_{1}\right)}{1+t_{1}^{\alpha-1}}\right| \\
& \leq \int_{0}^{\infty} \mid\left(\frac{G_{\alpha}\left(t_{2}, s\right)}{1+t_{2}^{\alpha-1}}-\frac{G_{\alpha}\left(t_{1}, s\right)}{1+t_{1}^{\alpha-1}}\right) \\
& \quad \times f\left(s, v(s), I^{\alpha} v(s)\right) \mid d s \\
& \leq \int_{0}^{\infty} \mid\left(\frac{K_{\alpha}\left(t_{2}, s\right)}{1+t_{2}^{\alpha-1}}-\frac{K_{\alpha}\left(t_{1}, s\right)}{1+t_{1}^{\alpha-1}}\right) \\
& +\left[\frac{\sum_{i=1}^{m-2} a_{i} \xi_{m-2}^{\alpha-1}}{\Gamma(\alpha) \Delta_{\alpha}}+\frac{\sum_{i=1}^{m-2} b_{i}}{\Delta_{\alpha}}\right\rfloor \\
& \quad \times \mid \frac{t_{2}^{\alpha-1}}{1+t_{2}^{\alpha-1}-\frac{t_{1}^{\alpha-1}}{1+t_{1}^{\alpha-1}} \mid} \\
& \times \int_{0}^{\infty}\left|f\left(s, v(s), I^{\alpha} v(s)\right)\right| d s
\end{aligned}
$$

In view of (37), by the similar process used in [20], we can easily prove that operator $T_{1}$ is equicontinuous. Similar process can be repeated for $T_{2}$; thus, $T$ is equicontinuous. On the other hand, $T B_{R}$ is uniformly bounded as $T B_{R} \subset B_{R}$. Therefore, $T$ is completely continuous operator. Hence, by Schauder fixed point theorem the boundary value problem (2) has at least one solution in $B_{R}$.

\section{An Example}

Consider the following boundary value problem on unbounded domain:

$$
\begin{aligned}
& D^{2.25} u(t)=\frac{\cos t \sin (|v(t)|)}{\left(1+t^{1.25}\right)(10+t)^{2}} \\
& +\frac{\left|\int_{0}^{\infty}(t-s)^{1.25} e^{-5 t} v(s) d s\right|}{\Gamma(2.25)\left(t^{2.25}+0.248 t^{3.5}\right)}+\frac{t^{2} e^{-t}}{1+t^{2}}, \\
& D^{2.5} v(t)=\frac{\sin t \arctan (|u(t)|)}{\left(1+t^{1.5}\right)(8+t)^{2}} \\
& +\frac{\left|\int_{0}^{\infty}(t-s)^{1.5} e^{-4 t} u(s) d s\right|}{\Gamma(2.5)\left(t^{2.5}+0.183 t^{4}\right)}+\frac{t^{4} e^{-t}}{1+t^{4}}, \\
& u(0)=u^{\prime}(0)=0, \quad v(0)=v^{\prime}(0)=0, \\
& D^{1.25} u(\infty)=\frac{1}{2} u\left(\frac{1}{4}\right)+\frac{1}{4} u(1)+\frac{1}{10} D^{1.25} u\left(\frac{1}{4}\right) \\
& +\frac{3}{10} D^{1.25} u(1) \\
& D^{1.5} v(\infty)=\frac{1}{5} v\left(\frac{1}{4}\right)+\frac{1}{10} v(1)+\frac{3}{11} D^{1.5} v\left(\frac{1}{4}\right) \\
& +\frac{1}{7} D^{1.5} v(1) \text {. }
\end{aligned}
$$

Here $t \in[0, \infty), \alpha=2.25, \beta=2.5, \xi_{1}=1 / 4, \xi_{2}=1, a_{1}=1 / 2$, $a_{2}=1 / 4, b_{1}=1 / 10, b_{2}=3 / 10, c_{1}=1 / 5, c_{2}=1 / 10, d_{1}=3 / 11$, and $d_{2}=1 / 7$. We have

$$
\begin{aligned}
f(t, x, y)= & \frac{\cos t \sin (|x|)}{\left(1+t^{1.25}\right)(10+t)^{2}} \\
& +\frac{e^{-5 t}|y|}{\left(t^{2.25}+0.248 t^{3.5}\right)}+\frac{t^{2} e^{-t}}{1+t^{2}}, \\
g(t, x, y)= & \frac{\sin t \arctan (|x|)}{\left(1+t^{1.5}\right)(8+t)^{2}} \\
& +\frac{e^{-4 t}|y|}{\left(t^{2.5}+0.183 t^{4}\right)}+\frac{t^{4} e^{-t}}{1+t^{4}} .
\end{aligned}
$$

For

$$
\begin{aligned}
& a(t)=\frac{1}{\left(1+t^{1.25}\right)(10+t)^{2}}, \\
& b(t)=\frac{e^{-5 t}}{\left(t^{2.25}+0.248 t^{3.5}\right)},
\end{aligned}
$$




$$
\begin{gathered}
\phi(t)=\varphi(t)=e^{-t}, \\
c(t)=\frac{1}{\left(1+t^{1.5}\right)(8+t)^{2}}, \\
d(t)=\frac{e^{-4 t}}{\left(t^{2.5}+0.183 t^{4}\right)},
\end{gathered}
$$

by direct calculation we obtain $\Delta_{\alpha}=0.342, \Delta_{\beta}=0.645, Q=$ 3.98 , and

$$
\begin{aligned}
& \int_{0}^{\infty}\left(1+t^{1.25}\right) a(t) d t+\frac{1}{\Gamma(2.25)} \\
& \times \int_{0}^{\infty}\left[\frac{t^{2.25}}{2.25}+B(2.25,2.25) t^{3.5}\right] b(t) d t \\
&= 0.17480<\frac{1}{Q}=0.25125, \\
& \int_{0}^{\infty}\left(1+t^{1.5}\right) c(t) d t+\frac{1}{\Gamma(2.5)} \\
& \quad \times \int_{0}^{\infty}\left[\frac{t^{2.5}}{2.5}+B(2.5,2.5) t^{4}\right] d(t) d t \\
&=0.20000<\frac{1}{Q}=0.25125 .
\end{aligned}
$$

Thus all the conditions of Theorem 8 are satisfied and the problem (38) has at least one solution.

\section{Conclusion}

In the current paper, we have studied the existence results for a coupled system of nonlinear fractional integrodifferential equations with $m$-point fractional boundary conditions on an unbounded domain. The result obtained in this paper is based on Schauder's fixed point theorem. In order to show the validity of the assumptions made in our result, we also include an illustrative example.

\section{Conflict of Interests}

The authors declare that there is no conflict of interests regarding the publication of this paper.

\section{References}

[1] R. Hilfer, Applications of Fractional Calculus in Physics, World Scientific Publishing, Singapore, 2000.

[2] A. A. Kilbas, H. M. Srivastava, and J. J. Trujillo, Theory and Applications of Fractional Differential Equations, vol. 204 of North-Holland Mathematics Studies, Elsevier Science B. V., Amsterdam, The Netherlands, 2006.

[3] V. Lakshmikantham, S. Leela, and J. V. Devi, Theory of Fractional Dynamic Systems, Cambridge Scientific Publishers, Cambridge, UK, 2009.
[4] B. Ahmad and J. J. Nieto, "Existence results for a coupled system of nonlinear fractional differential equations with threepoint boundary conditions," Computers \& Mathematics with Applications, vol. 58, no. 9, pp. 1838-1843, 2009.

[5] C. Bai and J. Fang, "The existence of a positive solution for a singular coupled system of nonlinear fractional differential equations," Applied Mathematics and Computation, vol. 150, no. 3, pp. 611-621, 2004.

[6] Y. Chen, D. Chen, and Z. Lv, "The existence results for a coupled system of nonlinear fractional differential equations with multi-point boundary conditions," Bulletin of the Iranian Mathematical Society, vol. 38, no. 3, pp. 607-624, 2012.

[7] M. Gaber and M. G. Brikaa, "Existence results for a coupled system of nonlinear fractional differential equation with fourpoint boundary conditions," ISRN Mathematical Analysis, vol. 2011, Article ID 468346, 14 pages, 2011.

[8] N. Khodabakhshi and S. M. Vaezpour, "Existence results for a coupled system of nonlinear fractional differential equations with boundary value problems on an unbounded domain," Electronic Journal of Qualitative Theory of Differential Equations, vol. 73, 15 pages, 2013.

[9] X. Su, "Boundary value problem for a coupled system of nonlinear fractional differential equations," Applied Mathematics Letters. An International Journal of Rapid Publication, vol. 22, no. 1, pp. 64-69, 2009.

[10] G. Wang, B. Ahmad, and L. Zhang, "A coupled system of nonlinear fractional differential equations with multipoint fractional boundary conditions on an unbounded domain," Abstract and Applied Analysis, vol. 2012, Article ID 248709, 11 pages, 2012.

[11] J. Wang, H. Xiang, and Z. Liu, "Positive solution to nonzero boundary values problem for a coupled system of nonlinear fractional differential equations," International Journal of Differential Equations, vol. 2010, Article ID 186928, 12 pages, 2010.

[12] W. Yang, "Positive solutions for a coupled system of nonlinear fractional differential equations with integral boundary conditions," Computers \& Mathematics with Applications, vol. 63, no. 1, pp. 288-297, 2012.

[13] C. Yuan, "Two positive solutions for $(n-1,1)$-type semipositone integral boundary value problems for coupled systems of nonlinear fractional differential equations," Communications in Nonlinear Science and Numerical Simulation, vol. 17, no. 2, pp. 930-942, 2012.

[14] A. Anguraj, P. Karthikeyan, and G. N'Guereata, "Nonlocal Cauchy problem for some fractional abstract integrodifferential equations in Banach spaces," Communications in Mathematical Analysis, vol. 6, no. 1, pp. 31-35, 2009.

[15] B. Ahmad and S. Sivasundaram, "Some existence results for fractional integro-differential equations with nonlinear conditions," Communications in Applied Analysis, vol. 12, no. 2, pp. 107-112, 2008.

[16] K. Balachandran and J. J. Trujillo, "The nonlocal Cauchy problem for nonlinear fractional integrodifferential equations in Banach spaces," Nonlinear Analysis: Theory, Methods and Applications, vol. 72, no. 12, pp. 4587-4593, 2010.

[17] D. Nazari and S. Shahmorad, "Application of the fractional differential transform method to fractional-order integrodifferential equations with nonlocal boundary conditions," Journal of Computational and Applied Mathematics, vol. 234, no. 3, pp. 883-891, 2010.

[18] J. Wang, X. Yan, X. H. Zhang, T. M. Wang, and X. Z. Li, "A class of nonlocal integrodifferential equations via fractional 
derivative and its mild solutions," Opuscula Mathematica, vol. 31, no. 1, pp. 119-135, 2011.

[19] Y. Liu, "Existence and unboundedness of positive solutions for singular boundary value problems on half-line," Applied Mathematics and Computation, vol. 144, no. 2-3, pp. 543-556, 2003.

[20] S. Liang and J. Zhang, "Existence of three positive solutions of $m$-point boundary value problems for some nonlinear fractional differential equations on an infinite interval," Computers \& Mathematics with Applications, vol. 61, no. 11, pp. 3343-3354, 2011. 


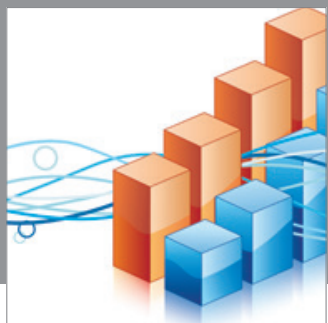

Advances in

Operations Research

mansans

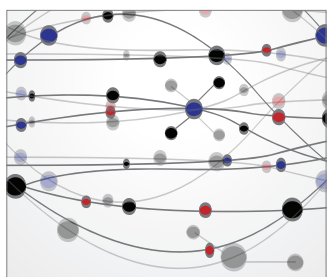

The Scientific World Journal
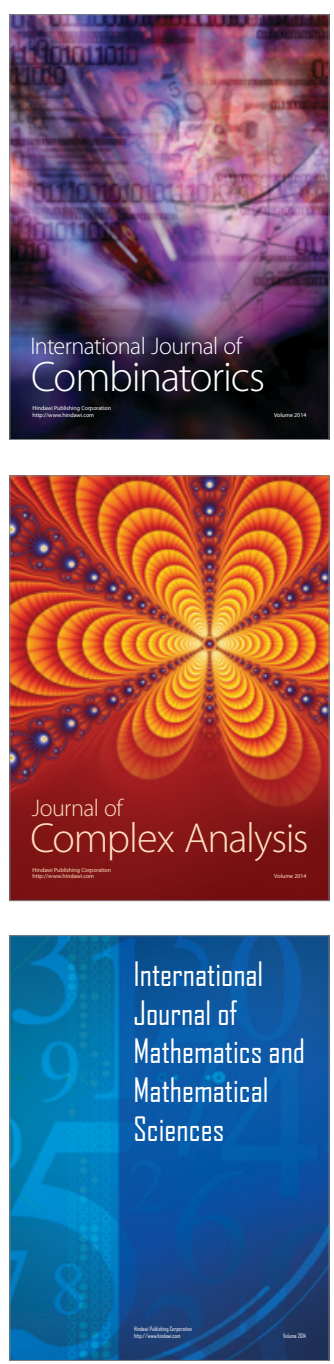
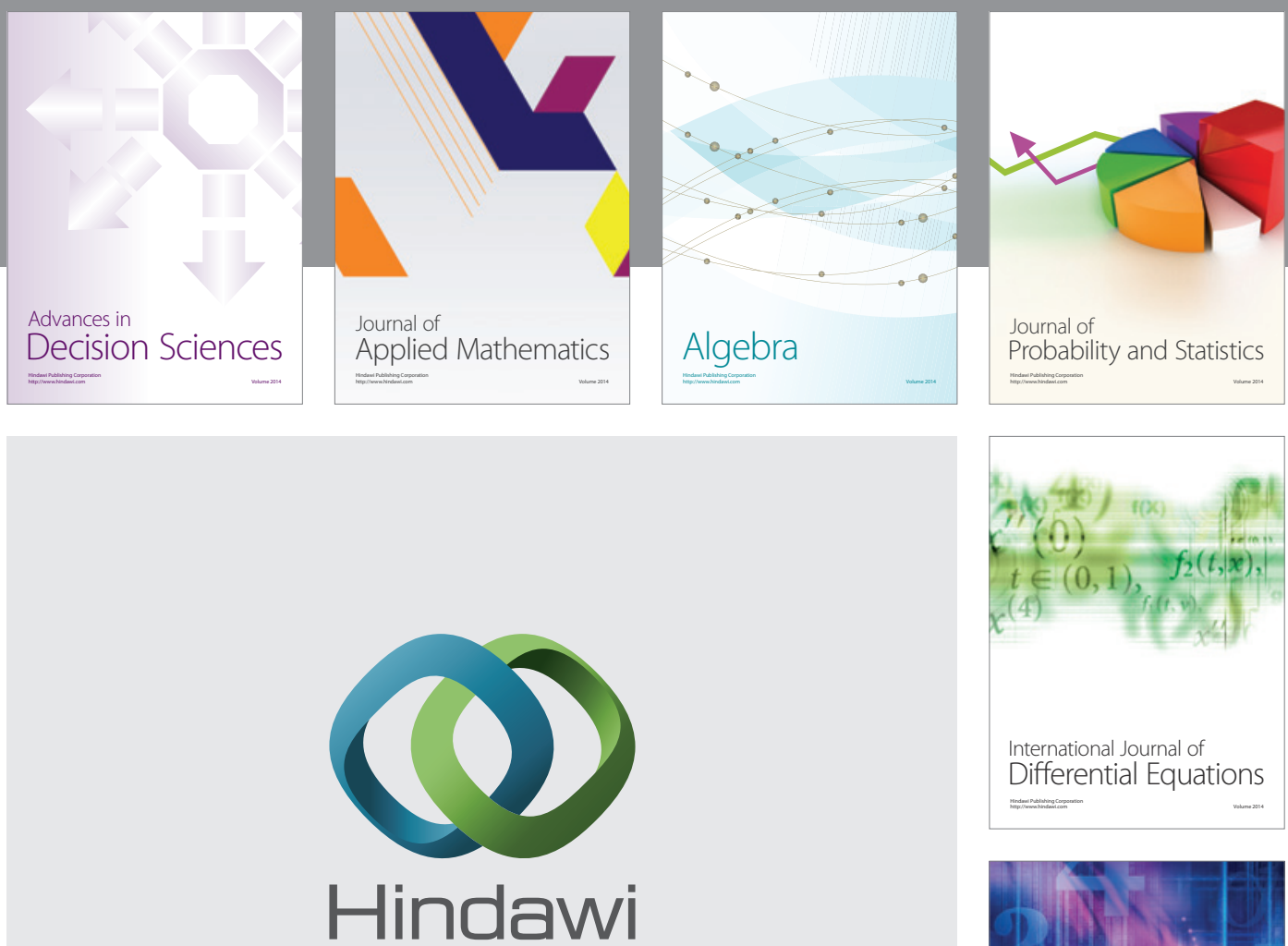

Submit your manuscripts at http://www.hindawi.com
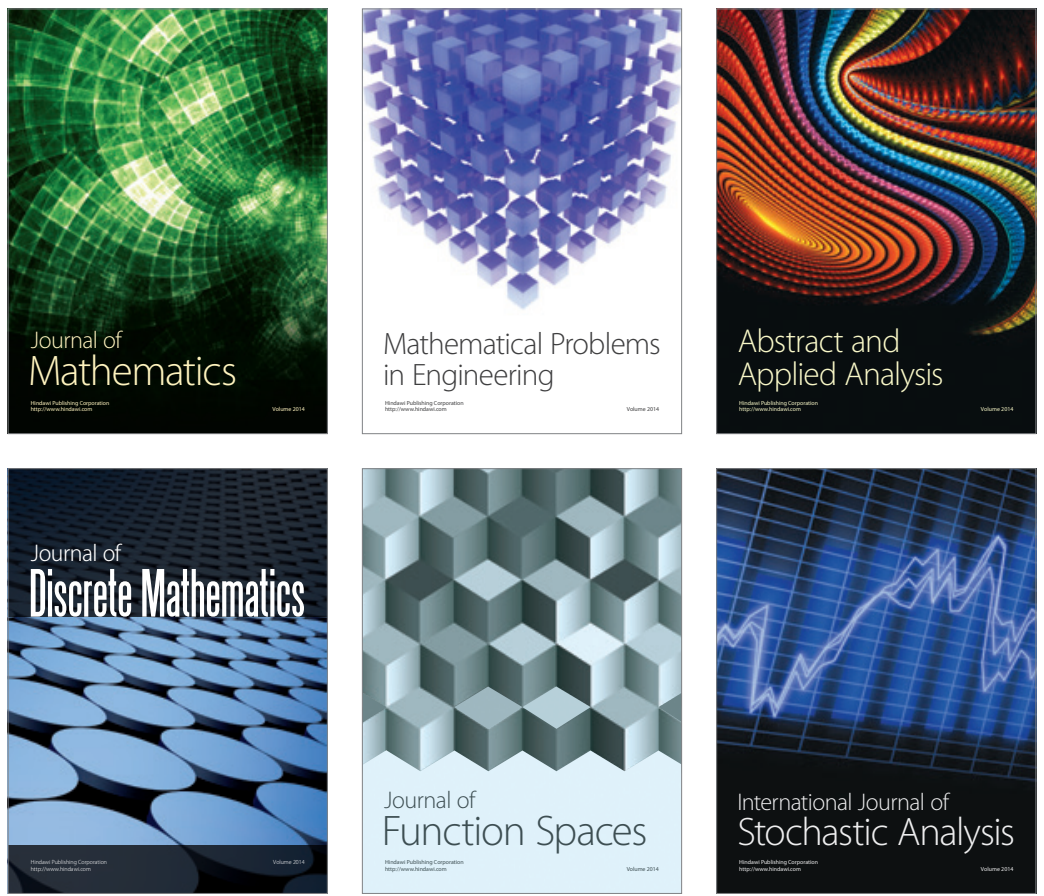

Journal of

Function Spaces

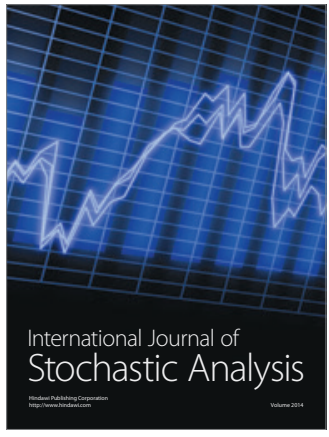

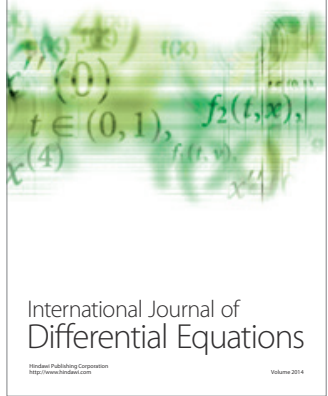
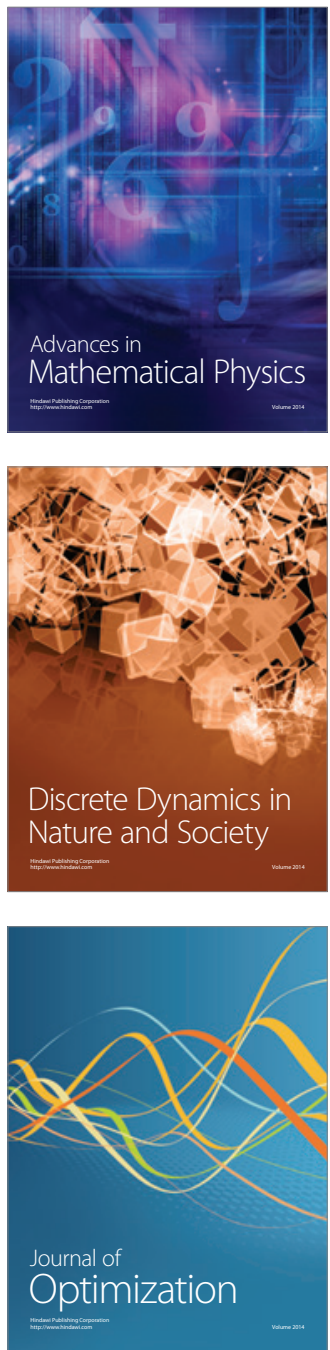